\title{
Desigualdades de Classe e Gênero e Saúde Mental nas Cidades
}

| ${ }^{1}$ Ana Bernarda Ludermir |

Resumo: O caráter social da doença mental se expressa objetivamente na sua distribuição desigual entre homens e mulheres e entre diferentes classes sociais. Essas desigualdades representam um persistente achado na literatura. Diversos autores têm encontrado alta prevalência dos Transtornos Mentais Comuns (TMC) em mulheres, nos excluídos do mercado formal de trabalho, nos indivíduos de baixa renda e nos de baixa escolaridade. Alguns estudos fundamentais para a visão contemporânea sobre as desigualdades de classe e gênero dos TMC são tratados neste artigo. As reflexões a respeito dos determinantes sociais da doença mental apontam desafios para a formulação de políticas públicas de saúde.

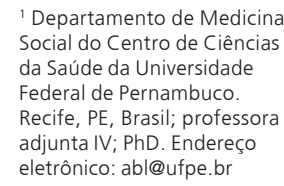

${ }^{1}$ Departamento de Medicina Social do Centro de Ciências da Saúde da Universidade Federal de Pernambuco. Recife, PE, Brasil; professora adjunta IV; PhD. Endereço eletrônico: abl@ufpe.br

Recebido em: 29/03/2008 Aprovado em: 08/08/2008 


\section{Introdução}

A doença mental caracteriza-se por uma determinação complexa que envolve dimensōes econômica, social, política e cultural, expressando-se diferentemente nas classes sociais e nas relações gênero.

A cidade moderna - caracterizada pela sociedade civil, pelo capitalismo e pela industrialização - é uma sociedade descontente no desejo de transcender as limitaçōes de caráter social e pessoal na sua totalidade (HELLER, 1993). A depressão tornou-se a "epidemia psíquica das sociedades democráticas" (ROUDINESCO, 2000) e será, segundo a Organização Mundial da Saúde (2000), a segunda causa de morbidade no mundo em 2020. Aliados à depressão, subsistem nas cidades a ansiedade e sintomas como insônia, fadiga, irritabilidade, esquecimento, dificuldade de concentração e queixas somáticas, definidos por Goldberg e Huxley (1992) como Transtornos Mentais Comuns (TMC).

Nos estudos de base populacional realizados em países industrializados, sua prevalência varia de 7\% a 30\% (GOLDBERG; HUXLEY, 1992). No Brasil, estudo realizado em 1994 revelou que 22,7\% da população adulta urbana de Pelotas-RS sofrem com os TMC (LIMA et al., 1996). Em Pernambuco, o estudo conduzido em adultos residentes na cidade de Olinda encontrou uma prevalência de 35\% (LUDERMIR; MELO FILHO, 2002). Frequentemente encontrados nas cidades, esses transtornos representam um alto custo social e econômico pois, incapacitantes, constituem causa importante de dias perdidos de trabalho, além de elevarem a demanda nos serviços de saúde (GOLDBERG; HUXLEY, 1992).

Para o movimento de sociologia urbana da Escola de Chicago das décadas de 20 e 30 do século XX, a vida das cidades per se produziria os sintomas psíquicos. Esta teoria vê a modernização como causa de estresse psíquico e baseia-se em duas premissas: 1) urbanização e industrialização trazem desorganização social e 2) o cotidiano rural é inerentemente mais saudável, do ponto de vista psicológico, que a vida nas cidades industrializadas. Nessa perspectiva romântica de mundo, o desenvolvimento tende ao retrocesso e a vida nas cidades significa a diminuição da liberdade e a destruição da espécie humana. Para esta escola, a impessoalidade da vida urbana, sua gama de estímulos, seu ritmo agitado, entre outras características, "criam doenças e vícios que tendem a destruir a comunidade" (PARK apud INKELES; SMITH, 1970, p. 82). 
Na América Latina, a industrialização poderia ter promovido a homogeneização socioeconômica e a eliminação dos contrastes colocados pela concentração de renda e de terra. Ao contrário disso, hoje se verifica que o desenvolvimento econômico não teve como desdobramento o desenvolvimento social; a heterogeneidade estrutural constituiu-se como uma das bases do caráter específico do desenvolvimento capitalista no continente. No Brasil, a industrialização, a urbanização acelerada e a expansão capitalista no campo desencadearam uma intensa migração interna, trazendo para as grandes cidades milhares de famílias à procura de emprego e melhores condiçôes de vida. Quanto mais a sociedade apregoa a emancipação, sublinhando a igualdade de todos perante a lei, mas se acentuam as diferenças e, em muitos casos, o local do migrante na estrutura social urbana está predeterminado por sua prévia posição de classe. A música de Chico Science (1994), “a cidade não pára/a cidade só cresce/o de cima sobe/e o de baixo desce”, explicita claramente o que Santos (1994) denomina de "involução metropolitana", com a expansão de empregos mal remunerados e a deterioração da qualidade de vida. As cidades brasileiras são marcadas por exclusão social, desemprego, prostituição e violência. Homens e mulheres expulsos da terra pelo latifúndio e pela introdução de tecnologia no campo passam a integrar outra paisagem, trocam o mocambo pela favela, vivem de lixo, pedem esmola, causam repugnância, atacam e roubam.

Este artigo discute as desigualdades de classe e gênero dos TMC e aponta alguns desafios para os formuladores de políticas de saúde mental ${ }^{1}$.

\section{Desigualdades de classe e saúde mental}

Um dos achados mais persistentes da epidemiologia psiquiátrica tem sido as desigualdades de classe dos TMC (BROWN; HARRIS, 1978; MURPHY et al., 1991; POWER et al., 1991; POWER; MANOR, 1992; STANSFELD; MARMOT, 1992; LUDERMIR; MELO FILHO, 2002; ALMEIDA-FILHO et al., 2004). A incorporação de conceitos das ciências sociais pelos epidemiologistas, entretanto, suscita alguns problemas teórico-metodológicos (DOHRENWEND, 1990). Estudos ingleses (POWER; MANOR, 1992; STANSFELD; MARMOT, 1992), por exemplo, utilizam a classificação social do Registro Geral, em que a população trabalhadora é agrupada pela ocupação em cinco "classes sociais" cuja amplitude se estende de profissionais e gerentes (I) até trabalhadores não 
qualificados (V). Essa classificação reduz "classe social" a um critério descritivo de setores populacionais, cujos limites são estabelecidos pela faixa salarial e pela posição no conjunto das ocupações (POSSAS, 1989; DOHRENWEND, 1990).

Numa perspectiva oposta, encontram-se aqueles que reconhecem "classe social" e "nível socioeconômico" como conceitos derivados de concepçôes teóricas distintas. Medidas de nível socioeconômico - como escolaridade, ocupação ou renda baseiam-se em prestígio e estratificação social. O conceito de classe social, por outro lado, recai sobre uma visão conflitual da sociedade, sendo as posições sociais definidas em termos da relação com os meios de produção (POSSAS, 1989; DOHRENWEND, 1990; WOHLFARTH, 1997).

As classes sociais não se referem a grupos empíricos; são forças atuantes com poder transformador da realidade social, não-operacionalizáveis. Para Possas (1989), as classes sociais, num plano complexo da realidade, determinam as condiçôes materiais de vida e a estrutura ocupacional - mediaçóes que explicam a magnitude e a distribuição das doenças nas populações. As relações sociais, determinadas por conflitos de classe no modo de produção capitalista, podem ser expressas por critérios de estratificação, mas a complexidade do conceito de classe não pode ser reduzida a elas.

\section{Educação}

Vários autores (LIMA et al., 1996; LUDERMIR; MELO FILHO, 2002; ARAYA et al., 2003) encontraram uma associação inversa entre anos de estudo e saúde mental. Para Brown e colaboradores (1986) a educação tem um efeito direto na saúde mental, pois aumenta a possibilidade de escolhas na vida e influencia aspiraçôes, auto-estima e aquisição de novos conhecimentos que podem motivar atitudes e comportamentos mais saudáveis.

Um aspecto ainda pouco explorado é o papel da escolaridade como modificador do efeito das associações de outros fatores com os transtornos mentais. Um estudo recente comparando Chile e Reino Unido - países com diferentes graus de desenvolvimento - demonstrou que o nível educacional foi responsável pelas diferentes prevalências de transtornos afetivos entre as chilenas e britânicas, sendo o maior risco encontrado nas chilenas de baixa escolaridade (ROJAS; ARAYA; LEWIS, 2005).

$\mathrm{O}$ acesso à escola reflete as circunstâncias sociais e materiais do início da vida, que são reproduzidas de uma geração para outra. A classe de origem determina o comportamento dos pais e influencia a permanência dos filhos na escola (RUTTER; 
MADGE, 1976). O nível de escolaridade, por sua vez, qualifica os indivíduos para certas ocupaçôes e interfere nas suas condições socioeconômicas futuras e inserção na estrutura ocupacional.

O sistema educacional brasileiro aprofunda as desigualdades de renda no país (URANI, 1995) e a evasão escolar está diretamente relacionada à atratividade do mercado de trabalho para crianças e adolescentes oriundas de famílias pobres. Estima-se que, entre 2005 e 2006, 400 mil menores do Semi-árido deixaram de freqüentar a escola pela necessidade precoce de trabalhar (ADITAL, 2008).

\section{Exclusão do mercado de trabalho: desemprego e informalidade}

Com altos custos sociais, o desemprego tem sido a maior questão político-econômica do mundo desde as últimas décadas do século XX. O desemprego estrutural, causado pelas novas formas de organização do trabalho e da produção - com a introdução de novas tecnologias como a robótica e a informática - afetou tanto os países desenvolvidos quanto os em desenvolvimento. Nos países latino-americanos, o desemprego estrutural veio acompanhado de um desemprego conjuntural, conseqüência da crise econômica que acometeu o continente (URANI, 1995).

A recente flexibilização das relações trabalhistas faz com que a divisão tradicional da força de trabalho em duas categorias - empregados e desempregados - não seja mais suficiente para descrever sua complexidade (VIRTANEN et al., 2003). Talvez a maior diferença entre a estrutura produtiva dos países desenvolvidos e dos em desenvolvimento esteja na proporção de trabalhadores fora do mercado formal de trabalho. Na América Latina, o exército industrial de reserva é constituído menos por desempregados, em senso estrito (SINGER, 1983; URANI, 1995), e mais por trabalhadores informais (serviçais domésticos, biscateiros e ambulantes), cujas atividades produtivas são executadas à margem da legislação trabalhista vigente (DESEP/CAP, 2004).

No Brasil, muitos trabalhadores formais que perderam seus empregos abandonaram o mercado de trabalho para se lançarem como autônomos no mercado de bens e serviços (URANI, 1995). Parte deles, provavelmente desencorajada pelas condições do mercado de trabalho, interrompeu a procura de emprego e "optou" pela informalidade como única forma de garantir remuneração (DIEESE, 1996). Apesar de esse tipo de trabalho ser característico dos países da América Latina (GUIMARÃES NETO; SOUZA, 1984; OLIVEIRA; ROBERTS, 1996), pouco se sabe sobre suas conseqüências psicológicas. 
Tem sido demonstrado que os desempregados têm pior saúde mental quando comparados aos que estão trabalhando (BROWN; HARRIS, 1978; BEBBINGTON et al., 1981; WARR, 1987; BARTLEY et al., 1992; JENKINS et al.; 1997; LIMA et al., 1996; LUDERMIR; LEWIS, 2003; ALMEIDA-FILHO et al., 2004). A saúde mental preexistente é um fator importante na determinação dos que conseguem e mantém os seus empregos - "the healthy worker effect" (LAHELMA, 1992); entretanto, estudos longitudinais de indivíduos saudáveis que vivenciaram o desemprego (WARR; JACKSON, 1985; HAMMARSTRÖM et al., 1988; BARTLEY et al., 1992; LAHELMA, 1992; GRAETZ, 1993; MORRELL et al., 1994; FERRIE et al., 1995) confirmam seus efeitos adversos, revertidos com a recuperação da saúde mental quando da volta ao emprego (WARR; JACKSON, 1985; LAHELMA, 1992; MORRELL et al., 1994).

O desemprego compreende a perda de um status e, freqüentemente, contém fases transitórias, de duração incerta (EZZY, 1993). Sua experiência varia com o significado que o indivíduo e a sociedade lhe atribuem (WARR, 1987; EZZY, 1993; GRAETZ, 1993). Assim, a relação do desemprego com os transtornos mentais apresenta claras desigualdades de gênero e de classes sociais. A associação entre desemprego e transtornos mentais é mais freqüente entre homens do que entre mulheres (RODGERS, 1991; LAHELMA, 1992; VIRTANEN et al., 2003). Estudos recentes (VIRTANEN et al., 2003) demonstram que há maior risco de depressão entre os desempregados de baixa renda, sugerindo que o efeito do desemprego na saúde mental pode ser atribuído a dificuldades financeiras (WEICH; LEWIS, 1998; VIRTANEN et al., 2003) para os já vulneráveis por sua posição de classe.

$\mathrm{O}$ mecanismo que liga o desemprego aos transtornos mentais ainda não está totalmente compreendido.

Warr (1987) descreve nove características consideradas como "vitaminas" para saúde mental em todos os tipos de ambiente, mas particularmente os do trabalho remunerado e do desemprego. São elas: 1) oportunidade para controle; 2) oportunidade para o uso de habilidades; 3) metas geradas externamente; 4) variedade; 5) previsibilidade; 6) disponibilidade de dinheiro; 7) segurança física; 8) oportunidade para contatos interpessoais e 9) valorização social. Sugere que os indivíduos podem moldar as influências ambientais de diferentes maneiras e explica, com seu modelo, os efeitos positivos observados com a recuperação do emprego, os negativos de um trabalho insatisfatório e as diferenças em saúde mental entre 
subgrupos de desempregados verificadas posterior e empiricamente por Rodgers (1991), Lahelma (1992) e Virtanen e colaboradores (2003).

Os mercados formal e informal representam destinos diferentes para aqueles que perderam os seus empregos e, recentemente, dois inquéritos epidemiológicos realizados no Brasil (SANTANA et al., 1997; LUDERMIR; LEWIS, 2005) encontraram associação entre a informalidade das relações de trabalho e a saúde mental das mulheres. No estudo de Ludermir e Lewis (2005), a prevalência de TMC entre as trabalhadoras informais $(54,4 \%)$ foi maior que a encontrada entre as desempregadas $(45,2 \%)$.

A informalidade apresenta algumas características que podem ser maléficas para a saúde psicológica, tais como: baixos níveis de poder de decisão e de controle pessoal (WARR, 1987; ROSENFIELD, 1989) sobre os vencimentos e a jornada de trabalho, determinados pela demanda do mercado, no caso dos autônomos, ou pela conveniência do empregador, para os subempregados. A incerteza sobre a situação de trabalho (WARR, 1987; VIRTANEN et al., 2003), os baixos salários, a ausência de benefícios sociais e de proteção da legislação trabalhista são provavelmente os responsáveis pelo desenvolvimento da ansiedade e depressão entre os trabalhadores informais.

\section{Renda}

A associação entre renda e TMC é uma das mais estudadas e consistentes na literatura nacional e internacional sobre os determinantes sociais da saúde mental (LIMA et al., 1996; WEICH; LEWIS, 1998; PATEL et al., 1999; LUDERMIR; MELO FILHO, 2002).

Alguns autores (BROWN; HARRIS, 1978; DRESSLER, 1985; CREED, 1993) sugerem que, para a saúde mental, os efeitos das dificuldades crônicas são mais importantes que os eventos vitais produtores de estresse. No entanto, Weich e Lewis (1998) encontraram resultados opostos e as dificuldades financeiras no momento das entrevistas mostraram-se associadas com a incidência e a prevalência dos TMC, enquanto a pobreza esteve apenas associada à manutenção dos episódios.

Para Lewis (1996) e Wilkinson (1996), a falta de dinheiro pode levar ao estresse e à insegurança, mecanismos psicológicos causadores dos TMC. Wilkinson (1997) sugere, no entanto, que pelo menos nos países desenvolvidos, as desigualdades de renda (pobreza relativa) comprometem mais a saúde do que as precárias condições 
de vida (pobreza absoluta), afirmando também que a tomada de consciência sobre as desigualdades socioeconômicas afeta a saúde mental. O autor afirma que

a associação da distribuição de renda com os homicídios, os crimes violentos, as mortes relacionadas ao uso de álcool e aos acidentes de trânsito reforçam a concepção de que as desigualdades de renda têm disseminado efeitos psicossociais (WILKINSON, 1996, p. 158).

\section{Associação entre os determinantes de classe dos TMC}

O nível de escolaridade - consolidado basicamente na adolescência (RUTTER; MADGE, 1976) - e a inserção produtiva e a renda - que representam a situação atual e podem variar ao longo da vida - expressam desigualdades de classe e, de maneiras diferentes, são determinantes da saúde mental.

Existe uma discussão (ARAYA et al., 2003) na literatura a respeito do efeito de cada um destes fatores sobre os transtornos mentais. Nos países europeus e norteamericanos, a associação entre renda e doença mental é recorrente (RODGERS, 1991; WEICH; LEWIS, 1998; KHAN et al., 2000), enquanto nos estudos realizados na América Latina, a escolaridade prevalece (BLUE, 2000; ARAYA et al., 2003).

Os fatores socioeconômicos têm sido freqüentemente associados entre si, levando alguns autores a propor índices compostos a fim de superar os efeitos da colinearidade - como o escore de pobreza de Weich e Lewis (1998) e a escala de classe social de Almeida-Filho e colaboradores (2004).

Distinguir entre si os fatores de risco para as desigualdades de classe dos TMC tem demonstrado ser, até o presente, uma discussão inconclusiva (ARAYA et al., 2003). Sua relevância, entretanto, se evidencia quando os resultados são tomados como subsídio para a formulação das políticas públicas (MARMOT et al., 1987).

\section{Desigualdades de gênero e saúde mental}

Especial atenção deve ser dada para as relações entre os TMC e as desigualdades de gênero, uma vez que um dos achados mais persistentes na literatura é que as mulheres têm uma prevalência de ansiedade e depressão duas a três vezes maior que os homens (PATEL et al., 1999; LUDERMIR; LEWIS, 2005). Vários autores têm estudado a associação das desigualdades de gênero e o sofrimento ou comprometimento mental, mediado pela baixa auto-estima das mulheres e seu 
menor controle sobre o meio ou contexto de vida (ROSENFIELD, 1989; JENKINS, 1985). Além disso, são parte das desigualdades de gênero tanto a construção do menor valor dos afazeres das mulheres na sociedade, quanto os desgastes e as percepções de pior saúde decorrentes, fazendo com que mulheres expressem mais facilmente seus sintomas, procurem mais os serviços de saúde que os homens e sejam efetivamente tratadas pelos profissionais de modo diverso (JENKINS, 1985; SCHRAIBER et al., 2005).

Adicionalmente, há que se considerar as mudanças atuais do mundo do trabalho e o conseqüente aumento do desemprego masculino versus o emprego das mulheres, configurando para elas uma somatória de atribuiçôes tradicionais de gênero e novas identidades, com jornadas ampliadas de trabalho (GIFFIN, 2002). Este exercício dos múltiplos papéis, acompanhado de antigos e novos modelos de subjetividade feminina, pode gerar conflitos e uma sensação de sobrecarga (role overload) levando à fadiga, ao estresse e a sintomas psíquicos (POWER et al., 1991; BARTLEY et al., 1992; MACRAN, 1993).

Tais mudanças agravam as já existentes assimetrias das relações de poder entre homens e mulheres. Isto gera situações de crise que resultam ou intensificam os conflitos domésticos entre parceiros afetivo-sexuais, podendo produzir a violência contra a mulher por seu parceiro íntimo (SCHRAIBER et al., 2005). A violência contra mulher engloba um amplo leque de abusos, geográfica e culturalmente particulares (WATTS; ZIMMERMAN, 2002), sendo não apenas manifestação das desigualdades de gênero, mas servindo para manter as injustiças contra as mulheres. A violência contra as mulheres é, na sua grande maioria, cometida por familiares ou pessoas próximas, tendo como principal agressor o parceiro íntimo (GARCIA-MORENO et al., 2006) que, em muitos casos, usa conscientemente a violência como mecanismo de subordinação, demonstrando e reforçando sua posição de poder enquanto chefe da casa ou da família (WATTS; ZIMMERMAN, 2002).

As dimensões física, sexual e psicológica mostram-se extremamente interligadas na violência cometida contra as mulheres. Revisões recentes mostram taxas de violência física cometida pelo parceiro íntimo ao menos uma vez na vida que variam de $10 \%$ a $56 \%$. Entre $10 \%$ e $30 \%$ das mulheres investigadas nesses estudos também referiram violência sexual (HEISE; GARCIA-MORENO, 2002; WATTS; ZIMMERMAN, 2002; KUMAR et al., 2005). Ellsberg (2000) demonstrou que 
94\% das mulheres vítimas de violência física também haviam sofrido insultos verbais e humilhações e 36\% referiram ser comumente forçadas a fazer sexo enquanto eram espancadas.

No Brasil, pesquisa realizada nacionalmente com mulheres de 15 anos ou mais, mostrou que $43 \%$ declararam ter sofrido violência praticada por um homem na vida, sendo que um terço relatou alguma forma de violência física, $13 \%$ sexual e 27\% psicológica (VENTURI et al., 2004). Da mesma pesquisa, revela-se que maridos, ex-maridos, namorados e ex-namorados foram os principais agressores, variando de $88 \%$ dos autores de tapas e empurrões a $79 \%$ dos perpetradores de relaçôes sexuais forçadas. O WHO Multi-country Study on Women's Health and Domestic Violence against women (GARCIA-MORENO et al., 2006), que comparou 15 regiōes de dez países, incluindo o Brasil, obteve, para a violência física por parceiro íntimo, ao menos uma vez na vida, uma prevalência de $27,2 \%$ na cidade de São Paulo e de 33,7\% para a Zona da Mata pernambucana. O estudo reiterou para os diferentes países a variação das prevalências, as elevadas taxas de violência e a violência por parceiro íntimo como a principal agressão contra as mulheres.

A violência tem um efeito devastador sobre a auto-estima da mulher, e muitos autores (ELLSBERG et al., 1999; NICOLAIDIS et al., 2004; KUMAR et al., 2005; RUIZ-PEREZ; PLAZAOLA-CASTAÑO, 2005; LUDERMIR et al., 2008) investigaram suas conseqüências para a saúde mental. Considerada pela Organização Mundial da Saúde (2000) como a principal causa, relativa a gênero, da depressão em mulheres, a violência cometida contra as mulheres provoca também ansiedade e aumento do uso de tranqüilizantes e antidepressivos (RUIZPEREZ; PLAZAOLA-CASTAÑO, 2005). O sofrimento mental duradouro é apontado como parte da "síndrome da mulher espancada" (McCAULEY et al., 1995), principal fator preditivo de consultas médicas e do uso de serviços de saúde de modo geral.

Para muitas mulheres, as conseqüências psicológicas da violência são ainda mais sérias que seus efeitos físicos (COKER et al., 2002; LUDERMIR et al., 2008). Estudos têm demonstrado que quanto mais severa a agressão, mas graves os impactos na saúde mental feminina (ELLSBERG et al., 1999; RUIZ-PEREZ; PLAZAOLACASTAÑO, 2005; LUDERMIR et al., 2008). As conseqüências de diferentes tipos de abuso e numerosos episódios parecem ser cumulativas, podendo persistir 
por muitos anos após o evento (ELLSBERG et al., 1999; RUIZ-PEREZ; PLAZAOLA-CASTAÑO, 2005). Seus efeitos são exacerbados pelo fato de o agressor ser um conhecido íntimo, o que aumenta a sensação de vulnerabilidade, traição e falta de esperança (HEISE et al., 1994).

A discriminação, os insultos verbais, os sentimentos de perda e os maus tratos, a degradação e a humilhação, características da violência contra a mulher, comprometem a auto-estima feminina e sua capacidade de reação e perpetuam o sentimento de subordinação (HEISE et al., 1994). A violência envolve a crença na onipotência do agressor (ELLSBERG et al., 1999) e gera sentimentos de derrota e perdas em vários níveis - a perda do sentido do que é próprio e do que é do outro, a perda da segurança nos relacionamentos e do sentimento de ser amada e cuidada (BROWN et al., 1995).

\section{Conclusão}

As desigualdades sociais podem ser analisadas segundo várias dimensões, incluindo classe social e gênero. Quando se perscrutam as causas estruturais das doenças mentais, na perspectiva de classe e gênero, percebem-se relações de subordinação e domínio, estabelecidas entre esses recortes sociais, que se expressam, sob a forma de sofrimento mental, de modo diferenciado entre esses grupos. Nessas relações estão imbricadas questões relativas a poder, privilégio e eqüidade.

Classe social e gênero representam conceitos distintos e sua associação com a saúde mental tem sido amplamente investigada. O núcleo essencial da concepção de causalidade da doença mental não se encontra numa determinada classe ou gênero, mas sim nas relaçóes estabelecidas entre classes e gêneros, caracterizadas pela opressão e subordinação de um grupo por outro.

As desigualdades sociais envolvem os principais sentimentos relacionados à depressão e a outros transtornos mentais, como humilhação, inferioridade, percepção de falta de controle sobre o meio e impotência. Observa-se, entretanto, que na perspectiva de classe e gênero, as necessidades sociais não são reconhecidas nos serviços de saúde, não se expressam nos diagnósticos formulados e a maioria dos profissionais não presta acolhimento adequado aos portadores de sofrimento mental. Gestores e profissionais da área de saúde devem considerar as desigualdades quando da formulação de políticas públicas, com o objetivo de prevenir os TMC. 


\section{Referências}

Agência de Informação Frei Tito para a América Latina. 132 milhões de crianças do mundo trabalham na agricultura. Disponível em: <http://www.adital.com.br/site/noticia.asp>. Acesso em: 19 jul. 2008.

ALMEIDA-FILHO, N. et al. Social inequality and depressive disorders in Bahia, Brazil: interactions of gender, ethnicity, and social class. Social Science \& Medicine, England, v.59, n.7, p.1339-1353, oct. 2004.

ARAYA, R. et al. Education and income: which is more important for mental health?, Journal Epidemiology Community Health, England, v.57, n.7, p.501-505, 2003.

BARTLEY, M.; OWEN, C. Relation between socioeconomic status, employment, and health during economic change, 1973-93. BMJ, England, v.313, n.7055, p.445-449, aug. 1996.

BARTLEY, M.; POPAY, J.; PLEWIS, I. Domestic conditions, paid employment and women's experience of ill-health. Sociology of Health and Illness, United States, v.14, n.3, p.313-343, 1992.

BEBBINGTON, P. et al. Epidemiology of mental disorders in Camberwell. Psychol Med, England, v.11, n.3 p.561-579, aug. 1981.

BLUE, I. Individual and contextual effects on mental health status in São Paulo, Brazil. Revista Brasileira de Psiquiatria, São Paulo, v.22, n.3, p.116-123, set. 2000.

BROWN, George W., HARRIS, Tirril. Social origins of depression: a study of psychiatric disorder in women. London: Tavistock, 1978. 399p.

BROWN, G.W.; HARRIS, T.O.; HEPWORTH, C. Loss and depression: a patient and nonpatient comparison. Psychol Med., England. v.25, n.1, p.7-21, 1995.

COKER, A. L. et al. Physical and mental health effects of intimate partner violence from men and women. American Journal of Preventive Medicine, Houston, v.24, n.4, p.260-268, 2002.

CREED, Francis. Life Events. In: BHUGRA, D.; LEFT, J. Principles of social psychiatry. Oxford: Blackwell Scientific, 1993. p.144-161.

Departamento Intersindical de Estatística e Estudos Sócio-Econômicos. Elementos para compreender a controvérsia sobre as taxas de desemprego no Brasil. São Paulo: SEADE/DIEESE, 1996.

Departamento Intersindical de Estatística e Estudos Sócio-Econômicos. Centro de Apoio. O trabalho informal no Brasil. Central Única dos Trabalhadores, Disponível em: < $\underline{\text { http://www.cut.org.br/ }}$ a50110.htm>. Acesso em: 9 de jul. 2004

DOHRENWEND, B.P. Socioeconomic status (SES) and psychiatric disorders: Are the issues still compelling?. Soc. Psychiatry Psychiatr. Epidemio., Germany, v.25, n.1, p.41-47, 1990.

DRESSLER, W.W. Extended family relationships, social support, and mental health in a southern black community. J. Health. Soc. Behav., United States, v.26, n..1, p.39-48, 1985. 
ELLSBERG, Mary Carol. Candies in hell: research and action on domestic violence against women in Nicaragua. Sweden: Umea, 2000. 95p.

ELLSBERG, M. et al. Domestic violence and emotional distress among Nicaraguan women: results from a population based study. Am. psychol, United States, v. 54, n.1, p.30-36, 1999.

EZZY, D. Unemployment and mental health: a critical review. Soc. Sci. Med., England, v.37, n1, p 41-52, 1993.

FERRIE, J.E., et al. Health effects of anticipation of job change and non-employment: longitudinal data from the Whitehall II study. BMJ, England, v.311, n.7015, p. 1264-1269, 1995.

GARCIA-MORENO, C. G. et al. Prevalence of intimate partner violence: findings from the WHO multi-country study on women's health and domestic violence. Lancet, England, v. 368, n.9543, p. 1260-1269, oct. 2006.

GIFFIN, K. Pobreza, desigualdade e equidade em saúde: considerações a partir de uma perspectiva de gênero transversal. Cad. Saúde Pública, Rio de Janeiro, vol. 18, p. 103-112. Suplemento.

GOLDBERG, David ; HUXLEY, Peter. Common mental disorders: a bio-social model. London; New York: Tavistock; Routledge, 1992. 194p.

GRAETZ, B. Health consequences of employment and unemployment: Longitudinal evidence for young men and women. Soc. Sci. Méd., England, v.36, n.6, p. 715-724, 1993.

GUIMARÃES NETO, Leonardo; SOUZA, Aldemir do Vale. A dinâmica de trabalho urbano no nordeste. In: CARVALHO, I. M. HAGUETE, T.M (Org.). Trabalho e condição de vida no Nordeste. São Paulo: HUCITEC, 1984. p. 14-41.

HAMMARSTRÖM, A.; JANLERT, U.; THEORELL, T. Youth employment and ill health: results from a 2-year follow-up study. Soc. Sci. Med., England, v. 26, n.10, p.1025-1033, 1988.

HARPHAM, T. Urbanization and mental health in developing countries: a research role for social scientists, public health professionals and social psychiatrists. Soc. Sci. Med., England, v. 39, n.2, p. 233-245, 1994.

HEISE, L.; PITANGUY, J.; GERMAIN, A. Violence against women: the hidden health burden. Soc. Sci. Med., England, v. 39, p. 233-245, 1994.

HEISE, L.; GARCIA-MORENO, C. Intimate partner violence. In: KRUG, E.G. et al. (Org.). World report on violence and health. Geneva: World Health Organization, 2002. p. 89-121.

HELLER, Agnes. Uma Teoria da História. Rio de Janeiro: Civilização Brasileira. 1993. 402p.

INKELES, A., SMITH, D. The fate of personal adjustment in the process of modernization. International Journal of Comparative Sociology, United States, v. 2, p. 81-113, 1970.

JENKINS, Rachel. Sex difference in minor psychiatric morbidity. Cambridge: Cambridge University Press, 1985. 53p. 
JENKINS, R. et. al. The National Psychiatric morbidity surveys of great Britain: initial findings from the household survey. Psychol Med, England, v. 27, n.4, p. 775-789, 1997.

KHAN, R.S. et al. State income inequality, household income, and maternal mental and physical health: cross sectional national survey. BMJ, England, v. 321, p.1311-15, 2000.

KUMAR, S., et al. Domestic violence and its mental health correlates in Indian women. $\mathrm{Br} J$ Psychiatry, England, v. 187, p. 62-67, 2005.

LAHELMA, E. Unemployment and mental well-being: elaboration of the relationship. Int. I. Health Serv, United States, v. 22, n.2, p. 261-274, 1992.

LEWIS, G. Depression and the public health. Int Rev Psychiatry, United States v. 8, n.4, p. 289294, 1996.

LIMA, M.S. et al. Stressful life events and minor psychiatric disorders: an estimate of the population attributable fraction in a Brazilian community-based study. Int I Psychiatry Med, United States, $\mathrm{n}$. 26, v.2, p 211-222, 1996.

LUDERMIR, A.B., LEWIS, G. Informal work and common mental disorders. Soc Psychiatry Psychiatr Epidemiol, Germany, v. 38, n.9, p. 485-489, 2003.

. Is there a gender difference on the association between informal work and common mental disorders? Soc Psychiatry Psychiatr Epidemiol, Germany, v. 40, n.8, p.622-627, 2005.

LUDERMIR, A.B.; MELO FILHO, D.A. Saúde mental, condiçôes de vida e estrutura ocupacional. Rev.Saúde Pública, São Paulo, v. 36, n. 2, p. 213-231, 2002.

LUDERMIR, A.B. et al. Violence against women by their intimate partner and common mental disorders. Soc Sci Med, England, v.66, n.4, p. 1008-1018, 2008.

MACHADO, L. M. V. The problems for woman-headed households in a low-income housing programme in Brazil. In: Moser, C. O. N.; Peake, L. (Org). Women, Human Settlement and Housing. London: Tavistock s, 1991, p. 55-69.

MACRAN, Susan. Role enhancement or role overload?: a review of research on the health consequence of women's domestic and paid work. London: Centre for Population Studies; London School of Hygiene and Tropical Medicine, 1993.27p.

MARMOT, M.G.; KOGEVINAS, M.; ELSTON, M.A. Social/economic status and disease, Annu. Rev Public Health, United States, v. 8: p. 111-135, 1987.

MCCAULEY, J.; KERN, D.E.; KOLODNER, K et al. The battering syndrome: prevalence and clinical characteristics of domestic violence in primary care internal medicine practices. Ann Intern Med., United States, v. 123, n.10, p. 737-746, 1995.

MORRELL, S.; TAYLOR, R.; QUINE, S. etal. A cohort study of unemployment as a cause of psychological disturbance in Australian youth. Soc Sci Med ${ }_{2}$ England, v. 38, n.11, p, 1553-1564, 1994. 
MOSER, Caroline O. N. Gender, planning and development: theory, practice and training. London: Routledge, $1993.306 \mathrm{p}$.

MURPHY, J.M. et al. Depression and anxiety in relation to social status. Arch Gen Psychiatry, United States, v. 48, n.3, p. 223-229, 1991.

NICOLAIDIS, C. et al. Violence, mental health, and physical symptoms in an academic internal medicine practice. J Gen Intern Med., United States, v. 19, n. 8, p. 819-827, 2004.

OLIVEIRA, Orlandina; ROBERTS, Bryan. Urban Development and Social Inequality in Latin America. In: . GUGLER, J. (Org). The urban transformation of the developing world. Great Britain: Oxford University Press, 1996. p. 253-314.

ORGANIZAÇÃO MUNDIAL DE SAÚDE. Women's mental health: an evidence based review. Geneva: WHO, 2000. 117p

PATEL, V. et al. Women, poverty and common mental disorders in four restructuring societies. Soc Sci Med, England, v. 49, n. 11, p.1461-1471, 1999.

POSSAS, Cristina. Epidemiologia e sociedade: heterogeneidade estrutural e saúde no Brasil. São Paulo: HUCITEC, 1989. 270p.

POWER, C.; MANOR, O. Explaining social class differences in psychological health among young adults: a longitudinal perspective. Soc Psychiatry Psychiatr Epidemiol, Germany, v.27 n.6, p. 284-291, 1992.

POWER, Chris; MANNOR, Orly; FOX, John. Health and class: the early years. London: Chapman and Hall, 1991. 216p.

RODGERS, B. Socio-economic status, employment and neurosis. Soc Psychiatry Psychiatr Epidemiol, Germany, v. 26, n.3, p. 104-114, 1991.

ROJAS, G., ARAYA, R., LEWIS, G. Comparing sex inequalities in common affective disorders across countries: Great Britain and Chile. Soc Sci Med, England, v. 60, n. 8, p.1.693-1.703, 2005.

ROSENFIELD, S. The effects of women's employment: personal control and sex differences in mental health. J Health Soc Behav, United States, v. 30, n.1, p. 77-91, 1989.

ROUDINESCO, Elizabeth. Por que a psicanálise? Rio de Janeiro: J. Zahar, 2000. 164p.

RUIZ-PEREZ, I.; PLAZAOLA-CASTANO, J. Intimate partner violence and mental health consequences in women attending family practice in Spain. Psychol Med, United States, n. 67, v. 5, p.791-797, 2005.

RUTTER, Michael; MADGE, Nicola. Cycles of Disadvantage: a review of research, London Heinemann, 1976. 413p.

SANTANA, V.S. et al. Informal jobs: another occupational hazard for woman's mental health. Int J Epidemiol, England, v. 26, n. 6, p. 1236-1242, 1997. 
SANTOS, Milton. Por uma economia politica da cidade. São Paulo: HUCITEC, 1994. 145p.

SCIENCE, Chico. A cidade. In: Chico Science e Nação Zumbi: da lama ao caos , CD, Rio de Janeiro: Sony Music, 1994.

SCHRAIBER, Lilia Blima et al. Violência dói e não é direito: a violência contra a mulher, saúde e os direitos humanos. São Paulo: Editora UNESP, 2005. 183p.

SINGER, Paul. Economia política da urbanização. São Paulo: Editora Brasiliense, 1983. 156p.

STANSFELD, S., MARMOT, M.G. Social class and minor psychiatric disorder in British civil servants: a validated screening survey using the general health questionnaire. Psychol Med, England, v. 22, n. 3, p. 739-749, 1992.

URANI, A. Crescimento e geração de emprego e renda no Brasil. Lua Nova: revista de cultura e política. São Paulo, v. 35, p. 5-37, 1995.

VENTURI, Gustavo; RECAMÁN, Marisol; OLIVEIRA, Suely. A mulher brasileira nos espaços público e privado. São Paulo: Editora Fundação Perseu Abramo, 2004. 220p.

VIRTANEN, P., LIUKKONEN, V., VAHTERA, J. et al. Health inequalities in the workforce: the labour market core-periphery structure. Int J Epidemiol, England, v. 32, n. 6, p. 1.015-1.021, 2003. WARR, Peter. Work, unemployment and mental health. Oxford: Clarendon Press, 1987. 328p.

WARR, P.; JACKSON, P. Factors influencing the psychological impact of prolonged unemployment and of re-employment. Psychol Med, England, v. 15, n. 4, p.795-807, 1985.

WATTS, C.; ZIMMERMAN, C. Violence against women: global scope and magnitude. Lancet, England, v. 359, n. 9313, p. 1232-1237, 2002.

WEICH, S.; LEWIS, G. Poverty, unemployment, and common mental disorders: population based cohort study. BMJ, England, v. 317, n.7151, p.115-119, 1998.

WILKINSON, Richard G. Unhealthy society: the afflictions of inequality. London; New York: Routledge, 1996. 225p.

. Socioeconomic determinants of health. Health inequalities: relative or absolute material standards? BMJ, England, v. 314, n. 7080, p.591-595, 1997.

WOHLFARTHT. Socioeconomic inequality and psychopathology: are socioeconomic status and social class interchangeable. Soc Sci Med, England, v. 45, n. 3, p. 399-410, 1997.

\section{Nota}

${ }^{1}$ Agradeço a Djalma Agripino de Melo Filho, Francisco Ludermir Ferreira e Rosa Ludermir, pelas importantes sugestôes na revisão deste artigo. 


\section{Class and gender inequalities and mental health in the cities}

The social nature of mental disorders is objectively apparent from its unequal distribution between men and women and between social classes. These inequalities are recurrent in the literature. Several authors have found high prevalence of Common Mental Disorders (CMD) among women, among the unemployed and informal workers, the low income and the low educated individuals. Some studies, fundamental for the contemporary view on the inequalities between class and gender of the CMD, are here presented. Considerations about the social determinants of mental illness point to some challenges for mental health planners.

> Key words: Mental health, common mental disorders, inequalities, class, gender. 